\title{
Online Supplement of \\ Optimal Uniform Pricing Strategy of a Service Firm When Facing Two Classes of Customers
}

\author{
by \\ Wenhui Zhou, Xiuli Chao, and Xiting Gong
}

This online supplement contains the proofs of all technical results. The proofs of Lemmas 2, and 4 are straightforward and are thus omitted.

\section{Proof of Lemma 1.}

Part (a) is obvious; hence we only provide the proof for (b). Since $W_{Q}(\lambda)$ is strictly increasing in $\lambda \in[0, \mu)$ with $W_{Q}(0)=0$, it follows that

$$
g(\lambda)=u_{1}(\lambda, p)-u_{2}(\lambda, p)=\left(w_{1}-w_{2}\right)-\left(d_{1}-d_{2}\right) W_{Q}(\lambda)
$$

is strictly decreasing in $\lambda \in[0, \mu)$ and it satisfies $g(0)>0, g(\lambda) \rightarrow-\infty$ as $\lambda \rightarrow \mu$. This shows that there exists a unique $\hat{\lambda} \in(0, \mu)$ satisfying $g(\hat{\lambda})=0, g(\hat{\lambda})>0$ on $\lambda<\hat{\lambda}$, and $g(\hat{\lambda})<0$ on $\lambda>\hat{\lambda}$. Solving $g(\lambda)=0$ with (2), we obtain (3).

\section{Proof of Theorem 1.}

(1) We first suppose $\Lambda_{1} \geq \lambda_{1}(p)$. We need to prove that the following is a Nash equilibrium for the customers: class- 1 customers enter with probability $\lambda_{1}(p) / \Lambda_{1}$, and no class- 2 customers enter the system. To this end, we first assume that class-1 customers follow the said strategy, i.e., class-1 customers enter the system at rate $\lambda_{1}(p)$. Then, it follows from $u_{2}\left(\lambda_{1}(p), p\right) \leq u_{1}\left(\lambda_{1}(p), p\right)=0$ that it is optimal for class- 2 customers not to enter the system for service, since doing so can only lead to negative utility. Next, suppose class- 2 customers follow the strategy of not entering the system for service, the utility value of the class- 1 customer is $u_{1}(\lambda, p)$ (if to enter with probability $\lambda / \Lambda_{1}$ ) and 0 (if to not enter). Note that $u_{1}(\lambda, p)$ is positive on $\lambda<\lambda_{1}(p) / \Lambda_{1}$ and negative on $\lambda>\lambda_{1}(p) / \Lambda_{1}$, this shows that class- 1 customers enter the system with probability $\lambda_{1}(p) / \Lambda_{1}$.

Next we consider $\lambda_{2}(p) \leq \Lambda_{1} \leq \lambda_{1}(p)$. We need to show that the following is an equilibrium: Class- 1 customers enter the system but no class- 2 customers enter the system. First suppose class- 2 customers follow the said strategy, if class- 1 customers enter the system with probability $\lambda / \Lambda_{1}$ with $\lambda \leq \Lambda_{1}$, then their utility value is $u_{1}(\lambda, p)$; and if they do not enter, then their utility value is 0 . Since $u_{1}(\lambda, p)$ is strictly decreasing in $\lambda$, we have $u_{1}(\lambda, p) \geq u_{1}\left(\Lambda_{1}, p\right)>u_{1}\left(\lambda_{1}(p), p\right)=0$. This shows that all customers will enter the system hence each class- 1 customer enters with probability 
1. On the other hand, suppose that class-1 customers follow the strategy of entering the system with probability 1 , then the utility value of class- 2 customers is $u_{2}\left(\lambda+\Lambda_{1}, p\right)$ (if to entering with probability $\left.\lambda / \Lambda_{2}\right)$ ) and 0 (if to not enter). Since $\lambda_{2}(p)$ is strictly decreasing in $p$ and $u_{2}(\lambda, p)$ is strictly decreasing in $\lambda$, it follows from $\Lambda_{1} \geq \lambda_{2}(p)$ that

$$
u_{2}\left(\lambda+\Lambda_{1}, p\right) \leq u_{2}\left(\Lambda_{1}, p\right) \leq u_{2}\left(\lambda_{2}(p), p\right)=0 .
$$

Thus, it is optimal for class-2 customers to not enter the system.

(2) Suppose $\Lambda_{1}<\lambda_{2}(p)$, we consider two subcases. First, $\lambda_{2}(p)<\Lambda_{1}+\Lambda_{2}$. We show that, in this subcase the Nash equilibrium is that class- 1 customers enter the system with probability 1 and class- 2 customers enter the system with probability $\left(\lambda_{2}(p)-\Lambda_{1}\right) / \Lambda_{2}$. Suppose class- 2 customers follow this strategy, we need to prove that the class- 1 customers enter the system with probability 1. Since for the dominating customers case we have $\lambda_{1}(p) \geq \lambda_{2}(p)$, it follows that when class-1 customers enter with probability 1 we have

$$
u_{1}\left(\left(\lambda_{2}(p)-\Lambda_{1}\right)+\Lambda_{1}, p\right)=u_{1}\left(\lambda_{2}(p), p\right) \geq u_{1}\left(\lambda_{1}(p), p\right)=0 .
$$

This shows that class- 2 customers also enter the system with probability 1 . Next, suppose class1 customers follow the prescribed strategy, then if customer- 2 customers enter the system with probability $\lambda / \Lambda_{2}$, then by $u_{2}\left(\lambda_{2}(p), p\right)=0$ we conclude that the utility value of class- 2 customers is negative when $\lambda>\lambda_{2}(p)-\Lambda_{1}$ and nonnegative otherwise. This shows that in this subcase the class- 2 customers enter the system with probability $\left(\lambda_{2}(p)-\Lambda_{1}\right) / \Lambda_{2}$.

We finally consider the subcase that $\lambda_{2}(p) \geq \Lambda_{1}+\Lambda_{2}$. We need to show that the Nash equilibrium for this case is that, all customers, class- 1 and class- 2 , join the system with probability 1 . Again, first suppose class- 1 customers follow this strategy, then when class- 2 customers join with probability 1 , their expected utility is $u_{2}(\Lambda, p) \geq u_{2}\left(\lambda_{2}(p), p\right)=0$. This shows that class 2 customers join the system as well. On the other hand, if class- 2 customers follow the prescribed strategy, then the utility value of class- 1 customers when entering with probability 1 is

$$
u_{1}\left(\Lambda_{1}+\Lambda_{2}, p\right) \geq u_{1}\left(\lambda_{2}(p), p\right) \geq u_{2}\left(\lambda_{2}(p), p\right)=0 .
$$

Thus class- 1 customers enter the system with probability 1 as well.

\section{Proof of Lemma 3.}

We only prove part (a), since part (b) can be similarly proved. Note that $w_{i}=v_{i}-d_{i} / \mu$. Then, $\hat{p}=\left(v_{2} d_{1}-v_{1} d_{2}\right) /\left(d_{1}-d_{2}\right)=\left(w_{2} d_{1}-w_{1} d_{2}\right) /\left(d_{1}-d_{2}\right)$. From (3) and (4), it is easy to verify that $\hat{\lambda}=\lambda_{1}(\hat{p})=\lambda_{2}(\hat{p})$. Since $\lambda_{i}(p)$ is strictly decreasing in $p, \lambda_{i}(p)>\hat{\lambda}$ when $p<\hat{p}$ while $\lambda_{i}(p)<\hat{\lambda}$ when $p>\hat{p}, i=1,2$. Thus, to complete the proof for part (a), it remains to show $\lambda_{1}(p)<\lambda_{2}(p)$ when $p<\hat{p}$ and $\lambda_{1}(p)>\lambda_{2}(p)$ when $p>\hat{p}$. In what follows, we only prove the result when $p<\hat{p}$, and the result when $p>\hat{p}$ can be similarly proved. 
When $p<\hat{p}$, since $\lambda_{2}(p)>\hat{\lambda}$, it follows from Lemma 1 that $u_{1}\left(\lambda_{2}(p), p\right)<u_{2}\left(\lambda_{2}(p), p\right)$. From the definition of $\lambda_{i}(p)$, we have $u_{1}\left(\lambda_{1}(p), p\right)=u_{2}\left(\lambda_{2}(p), p\right)=0$. Thus, $u_{1}\left(\lambda_{2}(p), p\right)<u_{1}\left(\lambda_{1}(p), p\right)$. Since $u_{1}(\lambda, p)$ is strictly decreasing in $\lambda$, we obtain $\lambda_{1}(p)<\lambda_{2}(p)$. The proof is complete.

\section{Proof of Theorem 2.}

(1) We first prove that, when $\Lambda_{1} \geq \lambda_{1}(p)$, the Nash equilibrium is, class-1 customers enter the system at rate $\lambda_{1}(p)$, and no class- 2 customers enter the system. Since $p \geq \hat{p}$, by Lemma 3 we have $\hat{\lambda} \geq \lambda_{1}(p) \geq \lambda_{2}(p)$. When class- 1 customers follow the said strategy, by the monotonicity of $u_{2}(\lambda, p)$ and the definition of $\lambda_{1}(p)$ and $\lambda_{2}(p)$, if class-2 customers join the system at rate $\lambda \leq \Lambda_{2}$, then

$$
u_{2}\left(\lambda_{1}(p)+\lambda, p\right) \leq u_{2}\left(\lambda_{1}(p), p\right) \leq u_{2}\left(\lambda_{2}(p), p\right)=0 .
$$

This shows that $\lambda=0$ and no class- 2 customer will enter the system. On the other hand, if class- 2 customers follow the prescribed strategy of not entering the system, then it follows from the monotonicity of $u_{1}(\lambda, p)$ and $u_{1}\left(\lambda_{1}(p), p\right)=0$ that class- 1 customers enter the system at rate $\lambda_{1}(p)$.

We next prove that, when $\lambda_{2}(p) \leq \Lambda_{1}<\lambda(p)$, the Nash equilibrium is, class-1 customers enter the system at rate $\Lambda_{1}$ and class- 2 customers do not enter the system. First suppose that class- 2 customers follow the said strategy. Since $\Lambda_{1}<\lambda_{1}(p)$, we have $u_{1}\left(\Lambda_{1}, p\right)>u_{1}\left(\lambda_{1}(p), p\right)=0$. Hence class- 1 customers enter the system at rate $\Lambda_{1}$. On the other hand, given that class- 1 customers follow the prescribed strategy, then from the monotonicity of $u_{2}(\lambda, p)$ in $\lambda$, we obtain $u_{2}\left(\Lambda_{1}, p\right) \leq$ $u_{2}\left(\lambda_{2}(p), p\right)=0$. This shows that class-2 customers do not enter the system.

(2) We need to prove that, the Nash equilibrium is, class-1 customers enter the system at rate $\Lambda_{1}$ and class- 2 customers enter at rate $\min \left\{\lambda_{2}(p)-\Lambda_{1}, \Lambda_{2}\right\}$. We consider two subcases. First, suppose $\lambda_{2}(p)<\Lambda_{1}+\Lambda_{2}$. When class-2 customers follow the prescribed strategy of entering the system at rate $\lambda_{2}(p)-\Lambda_{1}$, by Lemma 3 we have $\lambda_{1}(p) \geq \lambda_{2}(p)$. From the monotonicity of $u_{1}(\lambda, p)$ we have

$$
u_{1}\left(\left(\lambda_{2}(p)-\Lambda_{1}\right)+\Lambda_{1}, p\right)=u_{1}\left(\lambda_{2}(p), p\right) \geq u_{1}\left(\lambda_{1}(p), p\right)=0 .
$$

This shows that class- 1 customers enter the system at rate $\Lambda_{1}$. On the other hand, suppose class- 1 customers follow the strategy of entering the system at rate $\Lambda_{1}$, then by $u_{2}\left(\lambda_{2}(p), p\right)=0$ and the monotonicity of $u_{2}(\lambda, p)$ in $\lambda$, we conclude that class- 2 customers enter the system at rate $\lambda_{2}(p)-\Lambda_{1}$.

Now suppose $\lambda_{2}(p) \geq \Lambda_{1}+\Lambda_{2}$. If class-2 customers follow the said strategy, then by the monotonicity of $u_{1}(\lambda, p)$ in $\lambda$ and by Lemma 3 we have $\lambda_{1}(p) \geq \lambda_{2}(p)$, thus

$$
u_{1}\left(\Lambda_{1}+\Lambda_{2}, p\right) \geq u_{1}\left(\lambda_{2}(p), p\right) \geq u_{1}\left(\lambda_{1}(p), p\right)=0 .
$$

This shows that class-1 customers enter the system at rate $\Lambda_{1}$. On the other hand, suppose that class-1 customers follow the prescribed strategy. Since $u_{2}\left(\Lambda_{1}+\Lambda_{2}, p\right) \geq u_{2}\left(\lambda_{2}(p), p\right)=0$, we conclude that class- 2 customers enter the system at rate $\Lambda_{2}$. 
(3) If $p<\hat{p}$, then $\hat{\lambda}<\lambda_{1}(p)<\lambda_{2}(p)$ by Lemma 3. We first show that, when $\Lambda_{2} \geq \lambda_{2}(p)$, the Nash equilibrium is, class- 1 customers do not enter the system, and class- 2 customers enter the system at rate $\lambda_{2}(p)$. First suppose class-2 customers follow the said strategy, from the monotonicity of $u_{1}(\lambda, p)$, we have $u_{1}\left(\lambda_{2}(p), p\right)<u_{1}\left(\lambda_{1}(p), p\right)=0$, this shows that class- 1 customers do not enter the system. Next, if class- 1 customers do not enter the system, then it follows from $u_{2}\left(\lambda_{2}(p), p\right)=0$ that class-2 customers enter the system at rate $\lambda_{2}(p)$.

We next show that, when $\lambda_{1}(p) \leq \Lambda_{2} \leq \lambda_{2}(p)$, the Nash equilibrium is, class-1 customers do not enter the system and class- 2 customers enter the system at rate $\Lambda_{2}$. Suppose class- 1 customers follow the said strategy. By the monotonicity of $u_{2}(\lambda, p)$ in $\lambda$, we have $u_{2}\left(\Lambda_{2}, p\right)>u_{2}\left(\lambda_{2}(p), p\right)=0$, which means that class- 2 customers enter the system at rate $\Lambda_{2}$. On the other hand, if class-2 customers follow the prescribed strategy, then it follows from $u_{1}\left(\Lambda_{2}, p\right) \leq u_{1}\left(\lambda_{1}(p), p\right)=0$ that no class-1 customers will enter the system due to negative expected utility.

(4) We need to prove that the Nash equilibrium is, class-2 customers enter the system at rate $\Lambda_{2}$ and class- 1 customers enter the system at rate $\min \left\{\Lambda_{1}, \lambda_{1}(p)-\Lambda_{2}\right\}$. We consider two subcases. First, suppose $\Lambda_{1}+\Lambda_{2} \leq \lambda_{1}(p)$. If class-1 customers follow the said strategy, then it follows from $\lambda_{1}(p)<\lambda_{2}(p)$ that

$$
u_{2}\left(\Lambda_{1}+\Lambda_{2}, p\right) \geq u_{2}\left(\lambda_{1}(p), p\right)>u_{2}\left(\lambda_{2}(p), p\right)=0 .
$$

This implies that class- 2 customers enter the system at rate $\Lambda_{2}$. On the other hand, when class2 customers follow their prescribed strategy, then the expected utility of class-1 customers when entering at rate $\Lambda_{1}$ is $u_{1}\left(\Lambda_{1}+\Lambda_{2}, p\right) \geq u_{1}\left(\lambda_{1}(p), p\right)=0$. This shows that, class- 1 customers enter the system at rate $\Lambda_{1}$.

Second, suppose $\Lambda_{1}+\Lambda_{2}>\lambda_{1}(p)$. If class-1 customers follow the strategy of entering the system at rate $\lambda_{1}(p)-\Lambda_{2}$, then it follows from

$$
u_{2}\left(\left(\lambda_{1}(p)-\Lambda_{2}\right)+\Lambda_{2}\right)=u_{2}\left(\lambda_{1}(p), p\right)>u_{2}\left(\lambda_{2}(p), p\right)=0
$$

that class-2 customers enter the system at rate $\Lambda_{2}$. Similarly, if class- 2 customers follow the prescribed strategy, then it follows from $u_{1}\left(\lambda_{1}(p), p\right)=0$ and $\Lambda_{1}>\lambda_{1}(p)-\Lambda_{2}$ that class- 1 customers enter the system at rate $\lambda_{1}(p)-\Lambda_{2}$, since entering at a higher rate would result in negative utility. This completes the proof of the theorem.

\section{Proof of Proposition 1.}

We prove the result by analyzing the optimal price under different optimal customer bases being served. First, if under the optimal price $p^{*}$ only class- $i$ customers are served, then $p^{*}$ must be no less than $p_{i}^{*}$. This is because for a given service price $p$, the actual arrival rate of class- $i$ customers is $\min \left\{\Lambda_{i}, \lambda_{i}(p)\right\}$ and the firm's expected revenue is $\min \left\{p \Lambda_{i}, p \lambda_{i}(p)\right\}=\min \left\{p \Lambda_{i}, \pi_{i}(p)\right\}$. As $\pi_{i}(p)$ is strictly concave and $p_{i}^{*}=\arg \max _{p \geq 0} \pi_{i}(p)$, we conclude that $\min \left\{p \Lambda_{i}, \pi_{i}(p)\right\}$ is strictly increasing on $p \leq p_{i}^{*}$. This proves that the firm's optimal price in this case must be at least $p_{i}^{*}$. 
Second, if under the optimal price $p^{*}$ both classes of customers are served, then in this case $p^{*}$ must be no less than $\min \left\{p_{1}^{*}, p_{2}^{*}\right\}$. To prove this, first note that for a given service price $p$, the actual total arrival rate of two classes of customers is $\min \left\{\lambda_{1}(p), \lambda_{2}(p), \Lambda\right\}$. Since 1$)$ the total actual arrival rate must be less than $\min \left\{\lambda_{1}(p), \lambda_{2}(p)\right\}$ so both classes of customers are willing to enter the system, and 2) the total actual arrival rate must be less than the total potential rate $\Lambda$, we obtain that the firm's expected revenue is $\min \left\{\pi_{1}(p), \pi_{2}(p), \Lambda p\right\}$. As $\pi_{i}(p)$ is a strictly concave function and $p_{i}^{*}=\arg \max _{p \geq 0} \pi_{i}(p)$, the firm's optimal price must be at least $\min \left\{p_{1}^{*}, p_{2}^{*}\right\}$.

Because under the optimal price the firm serves either one class of customers or both classes of customers and we have shown for both cases the optimal price is at least $\min \left\{p_{1}^{*}, p_{2}^{*}\right\}$, the desired result follows.

\section{Proof of Lemma 5.}

We first prove $p_{1}^{*}>p_{2}^{*}$ and $\lambda_{1}^{*}>\lambda_{2}^{*}$. From (8), we have

$$
p_{i}^{*}=w_{i}\left(1-\frac{1+c_{v}^{2}}{\sqrt{\left(1+c_{v}^{2}\right)\left(\left(1+c_{v}^{2}\right)+2 \mu w_{i} / d_{i}\right)}-\left(1+c_{v}^{2}\right)}\right) .
$$

For the dominating case, we have $w_{1} \geq w_{2}>0, d_{1} \leq d_{2}$, and $d_{1}<d_{2}$ when $w_{1}=w_{2}$. Then, $w_{1} / d_{1}>w_{2} / d_{2}$. Hence, it follows from the above expression that $p_{1}^{*}>p_{2}^{*}$. In addition, from (7),

$$
\lambda_{i}^{*}=\arg \max _{0 \leq \lambda<\mu} \lambda p_{i}(\lambda)=\arg \max _{0 \leq \lambda<\mu} d_{i} \lambda\left(w_{i} / d_{i}-W_{Q}(\lambda)\right) .
$$

Since $w_{1} / d_{1}>w_{2} / d_{2}$, it follows from the above expression that $\lambda_{1}^{*}>\lambda_{2}^{*}$.

We next prove the remaining results in Lemma 5. Since $d_{1} \leq d_{2}$, it follows from Proposition 1 that $p_{1}(\lambda) \geq p_{2}(\lambda)$ and so $\tilde{\pi}_{1}(\lambda) \geq \tilde{\pi}_{2}(\lambda)$. Consequently, $\tilde{\pi}_{1}\left(\lambda_{1}^{*}\right) \geq \tilde{\pi}_{1}\left(\lambda_{2}^{*}\right) \geq \tilde{\pi}_{2}\left(\lambda_{2}^{*}\right)$; and it follows from Lemma 4 that $\underline{\lambda}_{2}^{*}=\lambda_{2}^{*}$.

Now suppose $0 \leq x \leq \underline{\lambda}_{1}^{*}$. By the definition of $\underline{\lambda}_{2}(x), \tilde{\pi}_{1}(x)=\tilde{\pi}_{2}\left(\underline{\lambda}_{2}(x)\right)$. Since $\tilde{\pi}_{2}\left(\underline{\lambda}_{2}(x)\right) \leq$ $\tilde{\pi}_{1}\left(\underline{\lambda}_{2}(x)\right)$, then $\tilde{\pi}_{1}(x) \leq \tilde{\pi}_{1}\left(\underline{\lambda}_{2}(x)\right)$. Since $\underline{\lambda}_{1}^{*} \leq \lambda_{1}^{*}$ by Lemma 4 and $\tilde{\pi}_{1}(x)$ is strictly increasing in $x$ when $0 \leq x \leq \lambda_{1}^{*}$, it follows that $\underline{\lambda}_{2}(x) \geq x$. Thus, when $0<x<\underline{\lambda}_{1}^{*}$, we have

$$
\underline{\lambda}_{2}^{\prime}(x)=\frac{\tilde{\pi}_{1}^{\prime}(x)}{\tilde{\pi}_{2}^{\prime}\left(\underline{\lambda}_{2}(x)\right)} \geq \frac{\tilde{\pi}_{1}^{\prime}(x)}{\tilde{\pi}_{2}^{\prime}(x)}=\frac{p_{1}(x)+x p_{1}^{\prime}(x)}{p_{2}(x)+x p_{2}^{\prime}(x)}>1,
$$

where the first inequality is from the concavity of $\tilde{\pi}_{2}(\cdot)$ and $\underline{\lambda}_{2}(x) \geq x$, and the second inequality holds since $p_{1}(x)>p_{2}(x)$ when $0<x<\underline{\lambda}_{1}^{*}$. Therefore, $\underline{\lambda}_{2}(x)-x$ is strictly increasing in $x$ when $0 \leq x \leq \underline{\lambda}_{1}^{*}$. Since $\underline{\lambda}_{2}(0)=0, \underline{\lambda}_{2}(x)>x$ when $0<x \leq \underline{\lambda}_{1}^{*}$; and in particular, $\underline{\lambda}_{2}\left(\underline{\lambda}_{1}^{*}\right)>\underline{\lambda}_{1}^{*}$. Note from Lemma 4 that $\underline{\lambda}_{2}\left(\underline{\lambda}_{1}^{*}\right)=\underline{\lambda}_{2}^{*}$. Then, $\underline{\lambda}_{2}^{*}>\underline{\lambda}_{1}^{*}$. The proof is complete. 


\section{Proof of Theorem 3.}

By Theorem 1, the equilibrium total arrival rate $\lambda(p)$ to the service firm can be written as

$$
\lambda(p)=\max \left\{\min \left\{\Lambda_{1}, \lambda_{1}(p)\right\}, \min \left\{\Lambda_{1}+\Lambda_{2}, \lambda_{2}(p)\right\}\right\} .
$$

Hence, the firm's revenue function $\pi(p)=p \lambda(p)$ is

$$
\pi(p)=\max \left\{\min \left\{\Lambda_{1} p, \pi_{1}(p)\right\}, \min \left\{\left(\Lambda_{1}+\Lambda_{2}\right) p, \pi_{2}(p)\right\}\right\} .
$$

Since $\pi_{i}(p)$ is strictly increasing in $p$ when $p \leq p_{i}^{*}, i=1,2$, it follows that

$$
\begin{aligned}
\max _{p \geq 0} \pi(p) & =\max \left\{\max _{p \geq 0}\left\{\min \left\{\Lambda_{1} p, \pi_{1}(p)\right\}\right\}, \max _{p \geq 0}\left\{\min \left\{\left(\Lambda_{1}+\Lambda_{2}\right) p, \pi_{2}(p)\right\}\right\}\right\} \\
& =\max \left\{\max _{p \geq p_{1}^{*}}\left\{\min \left\{\Lambda_{1} p, \pi_{1}(p)\right\}\right\}, \max _{p \geq p_{2}^{*}}\left\{\min \left\{\left(\Lambda_{1}+\Lambda_{2}\right) p, \pi_{2}(p)\right\}\right\}\right\} .
\end{aligned}
$$

Note that $\Lambda_{1} p$ is increasing in $p$ and $\pi_{1}(p)$ is strictly decreasing in $p$ when $p \geq p_{1}^{*}$. Then, when $\Lambda_{1} \geq \lambda_{1}^{*}, \max _{p \geq p_{1}^{*}}\left\{\min \left\{\Lambda_{1} p, \pi_{1}(p)\right\}\right\}=\pi_{1}\left(p_{1}^{*}\right)=p_{1}^{*} \lambda_{1}^{*}$; and when $\Lambda_{1} \leq \lambda_{1}^{*}, \min \left\{\Lambda_{1} p, \pi_{1}(p)\right\}$ achieves maximum at $\Lambda_{1}=\pi_{1}(p)$, or equivalently, at $p=p_{1}\left(\Lambda_{1}\right)$. By the definition of $\tilde{\pi}_{1}(\lambda)$, we have

$$
\max _{p \geq p_{1}^{*}}\left\{\min \left\{\Lambda_{1} p, \pi_{1}(p)\right\}\right\}=\tilde{\pi}_{1}\left(\min \left\{\Lambda_{1}, \lambda_{1}^{*}\right\}\right)
$$

Similarly,

$$
\max _{p \geq p_{2}^{*}}\left\{\min \left\{\left(\Lambda_{1}+\Lambda_{2}\right) p, \pi_{2}(p)\right\}\right\}=\tilde{\pi}_{2}\left(\min \left\{\Lambda_{1}+\Lambda_{2}, \lambda_{2}^{*}\right\}\right)
$$

Hence,

$$
\max _{p \geq 0} \pi(p)=\max \left\{\tilde{\pi}_{1}\left(\min \left\{\Lambda_{1}, \lambda_{1}^{*}\right\}\right), \tilde{\pi}_{2}\left(\min \left\{\Lambda_{1}+\Lambda_{2}, \lambda_{2}^{*}\right\}\right)\right\}
$$

In addition, it can be easily verified from the above procedure that: 1) $p^{*}=p_{1}^{*}$ if $\max _{p \geq 0} \pi(p)=$ $\tilde{\pi}_{1}\left(\lambda_{1}^{*}\right)$; 2) $p^{*}=p_{1}\left(\Lambda_{1}\right)$ if $\max _{p \geq 0} \pi(p)=\tilde{\pi}_{1}\left(\Lambda_{1}\right)$; 3) $p^{*}=p_{2}^{*}$ if $\max _{p \geq 0} \pi(p)=\tilde{\pi}_{2}\left(\lambda_{2}^{*}\right)$; and $p^{*}=$ $p_{2}\left(\Lambda_{1}+\Lambda_{2}\right)$ if $\max _{p \geq 0} \pi(p)=\tilde{\pi}_{2}\left(\Lambda_{1}+\Lambda_{2}\right)$.

Now we are ready to prove the theorem case by case. Note that by Lemma $2, \tilde{\pi}_{1}\left(\lambda_{1}^{*}\right) \geq \tilde{\pi}_{2}\left(\lambda_{2}^{*}\right)$ for the dominating customers case.

First, suppose $\Lambda_{1} \geq \lambda_{1}^{*}$. Then, $\max _{p \geq 0} \pi(p)=\tilde{\pi}_{1}\left(\lambda_{1}^{*}\right)$ by (11). Thus, the optimal price is $p_{1}^{*}$.

Second, suppose either $\underline{\lambda}_{1}^{*} \leq \Lambda_{1} \leq \lambda_{1}^{*}$ or $\Lambda_{1} \leq \underline{\lambda}_{1}^{*}$ and $\Lambda_{1}+\Lambda_{2} \leq \underline{\lambda}_{2}\left(\Lambda_{1}\right)$ are true. In both cases, by (11), we have

$$
\max _{p \geq 0} \pi(p)=\max \left\{\tilde{\pi}_{1}\left(\Lambda_{1}\right), \tilde{\pi}_{2}\left(\min \left\{\Lambda_{1}+\Lambda_{2}, \lambda_{2}^{*}\right\}\right)\right\} .
$$

When $\underline{\lambda}_{1}^{*} \leq \Lambda_{1} \leq \lambda_{1}^{*}$, from the definition of $\underline{\lambda}_{1}^{*}$, $\tilde{\pi}_{1}\left(\Lambda_{1}\right) \geq \tilde{\pi}_{2}\left(\underline{\lambda}_{1}^{*}\right)=\pi_{2}\left(p_{2}^{*}\right)$. When $0 \leq \Lambda_{1} \leq \underline{\lambda}_{1}^{*}$ and $\Lambda_{1}+\Lambda_{2} \leq \underline{\lambda}_{2}\left(\Lambda_{1}\right)$, it follows from Lemma 4 that $\Lambda_{1}+\Lambda_{2} \leq \lambda_{2}^{*}$. Then, $\tilde{\pi}_{2}\left(\min \left\{\Lambda_{1}+\Lambda_{2}, \lambda_{2}^{*}\right\}\right)=$ 
$\tilde{\pi}_{2}\left(\Lambda_{1}+\Lambda_{2}\right) \leq \tilde{\pi}_{2}\left(\underline{\lambda}_{2}\left(\Lambda_{1}\right)\right)=\tilde{\pi}_{1}\left(\Lambda_{1}\right)$. Thus, for both cases, $\max _{p \geq 0} \pi(p)=\tilde{\pi}_{1}\left(\Lambda_{1}\right)$, and the optimal price is $p_{1}\left(\Lambda_{1}\right)$.

Third, suppose $0 \leq \Lambda_{1} \leq \underline{\lambda}_{1}^{*}$ and $\Lambda_{1}+\Lambda_{2} \geq \lambda_{2}^{*}$. In this case, since $\underline{\lambda}_{1}^{*} \leq \lambda_{1}^{*}$, we have

$$
\tilde{\pi}_{1}\left(\min \left\{\Lambda_{1}, \lambda_{1}^{*}\right\}\right)=\tilde{\pi}_{1}\left(\Lambda_{1}\right) \leq \tilde{\pi}_{1}\left(\underline{\lambda}_{1}\right)=\tilde{\pi}_{2}\left(\lambda_{2}^{*}\right)=\tilde{\pi}_{2}\left(\min \left\{\Lambda_{1}+\Lambda_{2}, \lambda_{2}^{*}\right\}\right) .
$$

Then, by $(11), \max _{p \geq 0} \pi(p)=\tilde{\pi}_{2}\left(\lambda_{2}^{*}\right)$. Thus, the optimal price is $p_{2}^{*}$.

Finally, suppose $0 \leq \Lambda_{1} \leq \underline{\lambda}_{1}^{*}$ and $\underline{\lambda}_{2}\left(\Lambda_{1}\right) \leq \Lambda_{1}+\Lambda_{2} \leq \lambda_{2}^{*}$. In this case, we have

$$
\tilde{\pi}_{1}\left(\min \left\{\Lambda_{1}, \lambda_{1}^{*}\right\}\right)=\tilde{\pi}_{1}\left(\Lambda_{1}\right)=\tilde{\pi}_{2}\left(\underline{\lambda}_{2}\left(\Lambda_{1}\right)\right) \leq \tilde{\pi}_{2}\left(\Lambda_{1}+\Lambda_{2}\right)=\tilde{\pi}_{2}\left(\min \left\{\Lambda_{1}+\Lambda_{2}, \lambda_{2}^{*}\right\}\right)
$$

Then, by (11), $\max _{p \geq 0} \pi(p)=\tilde{\pi}_{2}\left(\Lambda_{1}+\Lambda_{2}\right)$. Consequently, the optimal price is $p_{2}(\Lambda)$.

Summarizing the above different cases and applying Theorem 1, we obtain Theorem 3.

\section{Proof of Proposition 2.}

1) According to Lemma 1 , it is easily seen that $\pi_{1}\left(p_{1}^{*}\right)>\pi_{2}\left(p_{2}^{*}\right)$. By the definition of $\underline{\lambda}_{1}^{*}$ and $\underline{\lambda}_{2}\left(\Lambda_{1}\right)$, it follows that

$$
R^{(I)}=\pi_{1}\left(p_{1}^{*}\right)>\pi_{1}\left(p_{1}\left(\Lambda_{1}\right)\right)=R^{(I I)}>\pi_{2}\left(p_{2}^{*}\right)=R^{(I I I)}>\pi_{2}\left(p_{2}\left(\Lambda_{1}+\Lambda_{2}\right)\right)=R^{(I V)} .
$$

2) By the definition of $\underline{\lambda}_{1}^{*}$, it follows that $\underline{\lambda}_{1}^{*} \leq \lambda_{1}^{*}$. Note that $\tilde{\pi}_{1}(\lambda)$ is strictly increasing in $\lambda$ when $0 \leq \lambda \leq \lambda_{1}^{*}$. Then,

$$
R^{(I I I)}=\pi_{2}\left(p_{2}^{*}\right)=\tilde{\pi}_{2}\left(\lambda_{2}^{*}\right)=\tilde{\pi}_{1}\left(\underline{\lambda}_{1}^{*}\right)>\tilde{\pi}_{1}\left(\Lambda_{1}\right)=\pi_{1}\left(p_{1}\left(\Lambda_{1}\right)\right)=R^{(V)} .
$$

The proof is complete.

\section{Proof of Lemma 6.}

We only prove $\hat{p} \geq p_{2}^{*}$ if $\hat{p} \geq p_{1}^{*}$, and the result that $\hat{p} \leq p_{1}^{*}$ if $\hat{p} \leq p_{2}^{*}$ can be proved similarly. Suppose $\hat{p} \geq p_{1}^{*}$. Then, $\pi_{1}(p)$ is strictly decreasing in $p$ when $p \geq \hat{p}$, and thus $\pi_{1}(p) \leq \pi_{1}(\hat{p})$ for any $p \geq \hat{p}$. In addition, from Lemma $3, \pi_{1}(\hat{p})=\pi_{2}(\hat{p})$ and $\lambda_{1}(p) \geq \lambda_{2}(p)$ for any $p \geq \hat{p}$. It follows that $\pi_{2}(p) \leq \pi_{2}(\hat{p})$ for any $p \geq \hat{p}$. Thus, by the definition of $p_{2}^{*}$, we obtain $p_{2}^{*} \leq \hat{p}$.

(a) Suppose $\hat{p} \leq \min \left\{p_{1}^{*}, p_{2}^{*}\right\}$. In this case, $\hat{\lambda} \geq \max \left\{\lambda_{1}^{*}, \lambda_{2}^{*}\right\}$, and from Lemma $3, p_{1}^{*} \lambda_{1}^{*}=$ $\pi_{1}\left(p_{1}^{*}\right) \geq \pi_{1}\left(p_{2}^{*}\right) \geq \pi_{2}\left(p_{2}^{*}\right)=p_{2}^{*} \lambda_{2}^{*}$. Thus, by Lemma $4, \underline{\lambda}_{2}^{*}=\lambda_{2}^{*}$. Now suppose $0 \leq x \leq \underline{\lambda}_{1}^{*}$. By the definition of $\underline{\lambda}_{2}(x), \tilde{\pi}_{1}(x)=\tilde{\pi}_{2}\left(\underline{\lambda}_{2}(x)\right)$. Since $\underline{\lambda}_{2}(x) \leq \lambda_{2}^{*} \leq \hat{\lambda}$ by Lemma 4 , it follows from Lemma 3 that $\tilde{\pi}_{2}\left(\underline{\lambda}_{2}(x)\right) \leq \tilde{\pi}_{1}\left(\underline{\lambda}_{2}(x)\right)$, and then, $\tilde{\pi}_{1}\left(\underline{\lambda}_{2}(x)\right) \geq \tilde{\pi}_{1}(x)$. Since $\underline{\lambda}_{1}^{*} \leq \lambda_{1}^{*}$ by Lemma 4 and $\tilde{\pi}_{1}(x)$ is strictly increasing in $x$ when $0 \leq x \leq \lambda_{1}^{*}$, it follows that $\underline{\lambda}_{2}(x) \geq x$. In particular, $\underline{\lambda}_{2}\left(\underline{\lambda}_{1}^{*}\right) \geq \underline{\lambda}_{1}^{*}$. Note from Lemma 4 that $\underline{\lambda}_{2}\left(\underline{\lambda}_{1}^{*}\right)=\underline{\lambda}_{2}^{*}$. Then, $\underline{\lambda}_{2}^{*} \geq \underline{\lambda}_{1}^{*}$. 
(b) Suppose $\hat{p} \geq \max \left\{p_{1}^{*}, p_{2}^{*}\right\}$. In this case, $\hat{\lambda} \leq \min \left\{\lambda_{1}^{*}, \lambda_{2}^{*}\right\}$, and from Lemma $3, \pi_{2}\left(p_{2}^{*}\right) \geq$ $\pi_{2}\left(p_{1}^{*}\right) \geq \pi_{1}\left(p_{1}^{*}\right)$. Thus, by Lemma $4, \underline{\lambda}_{1}^{*}=\lambda_{1}^{*}$. Since $\tilde{\pi}_{1}(\hat{\lambda})=\tilde{\pi}_{2}(\hat{\lambda})$, it is easily seen from the definition of $\underline{\lambda}_{i}(x)$ that $\hat{\lambda}=\underline{\lambda}_{1}(\hat{\lambda})=\underline{\lambda}_{2}(\hat{\lambda})$. Since $\underline{\lambda}_{2}(x)$ is increasing in $x$ by Lemma 4 , then $\underline{\lambda}_{2}(\hat{\lambda}) \leq \underline{\lambda}_{2}\left(\underline{\lambda}_{1}^{*}\right)=\underline{\lambda}_{2}^{*}$ and $\underline{\lambda}_{2}(x) \leq \underline{\lambda}_{2}(\hat{\lambda})=\hat{\lambda}$ when $0 \leq x \leq \hat{\lambda}$. In addition, when $0 \leq x \leq \hat{\lambda}$, we have $\tilde{\pi}_{2}\left(\underline{\lambda}_{2}(x)\right)=\tilde{\pi}_{1}(x) \geq \tilde{\pi}_{2}(x)$ by Lemma 3 , since $\tilde{\pi}_{2}(x)$ is increasing in $x$ when $0 \leq x \leq \lambda_{2}^{*}$, it follows that $\underline{\lambda}_{2}(x) \geq x$. Now assume $\hat{\lambda} \leq x \leq \underline{\lambda}_{2}^{*}$. Then, $\tilde{\pi}_{1}\left(\underline{\lambda}_{1}(x)\right)=\tilde{\pi}_{2}(x) \geq \tilde{\pi}_{1}(x)$ by Lemma 3 . Since $\tilde{\pi}_{1}(x)$ is strictly increasing in $x$ when $0 \leq x \leq \lambda_{1}^{*}$, it follows that $\underline{\lambda}_{1}(x) \geq x$. In particular, $\underline{\lambda}_{1}\left(\underline{\lambda}_{2}^{*}\right) \geq \underline{\lambda}_{2}^{*}$. Note from Lemma 4 that $\underline{\lambda}_{1}\left(\underline{\lambda}_{2}^{*}\right)=\underline{\lambda}_{1}^{*}$. Then, $\underline{\lambda}_{1}^{*} \geq \underline{\lambda}_{2}^{*}$.

(c) Suppose $p_{2}^{*}<\hat{p}<p_{1}^{*}$. In this case, $\lambda_{1}^{*}<\hat{\lambda}<\lambda_{2}^{*}$. Note from Lemma 4 that $0 \leq \underline{\lambda}_{2}^{*} \leq \lambda_{2}^{*}$ and $\tilde{\pi}_{2}\left(\underline{\lambda}_{2}^{*}\right)=\min \left\{\tilde{\pi}_{1}\left(\lambda_{1}^{*}\right), \tilde{\pi}_{2}\left(\lambda_{2}^{*}\right)\right\}>\tilde{\pi}_{2}(\hat{\lambda})$. Then, since $\tilde{\pi}_{2}(\lambda)$ is strictly increasing in $\lambda$ when $0 \leq \lambda \leq$ $\lambda_{2}^{*}$, we obtain $\hat{\lambda}<\underline{\lambda}_{2}^{*} \leq \lambda_{2}^{*}$. In addition, it follows from Lemma 4 that $\underline{\lambda}_{1}(\hat{\lambda})<\underline{\lambda}_{1}\left(\underline{\lambda}_{2}^{*}\right)=\underline{\lambda}_{1}^{*} \leq \lambda_{1}^{*}$. Now assume $0 \leq x \leq \underline{\lambda}_{1}(\hat{\lambda})$. Then, $0 \leq x \leq \lambda_{1}^{*}<\hat{\lambda}$ and so $\tilde{\pi}_{2}\left(\underline{\lambda}_{2}(x)\right)=\tilde{\pi}_{1}(x)>\tilde{\pi}_{2}(x)$ by Lemma 3. Since $\tilde{\pi}_{2}(x)$ is strictly increasing in $x$ when $0 \leq x \leq \lambda_{2}^{*}$, it follows that $\underline{\lambda}_{2}(x) \geq x$. In addition, from Lemma $4, \underline{\lambda}_{2}\left(\underline{\lambda}_{1}(\hat{\lambda})\right)=\hat{\lambda}$, so $\underline{\lambda}_{2}(x) \leq \hat{\lambda}$ when $0 \leq x \leq \underline{\lambda}_{1}(\hat{\lambda})$. The proof is complete.

For completeness, we present the optimal pricing decision for all the non-dominating customer case altogether in the following Theorem.

Theorem 5. (Optimal price for non-dominating customer case) When $d_{1}>d_{2}$, the optimal price of the firm $p^{E Q}$ and customers' equilibrium behavior $\left(\theta_{1}^{E Q}, \theta_{2}^{E Q}\right)$ are given in four exclusive regions of the system parameters as follows:

Case 1: $\hat{p} \leq \min \left\{p_{1}^{*}, p_{2}^{*}\right\}$. In this case, the optimal pricing strategy of the firm is given exactly in the same way as that in Theorem 3, and it is illustrated in Figure 4.

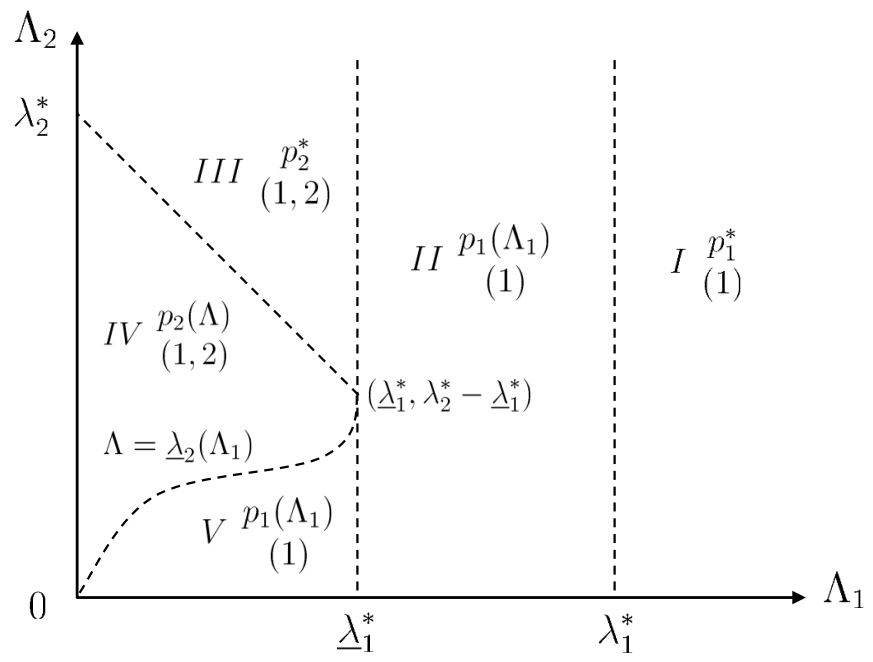

Figure 4: Optimal policy when $\hat{p} \geq \max \left\{p_{1}^{*}, p_{2}^{*}\right\}$ 
Case 2. $\hat{p} \geq \max \left\{p_{1}^{*}, p_{2}^{*}\right\}$. In this case, the optimal pricing strategy of the firm is given according to the following subcases, and it is illustrated in Figure 5:

(2-1) if $\Lambda_{2} \geq \underline{\lambda}_{2}^{*}$, then $p^{E Q}=\max \left\{p_{2}^{*}, p_{2}\left(\Lambda_{2}\right)\right\}$ and $\left(\theta_{1}^{E Q}, \theta_{2}^{E Q}\right)=\left(0, \min \left\{1, \lambda_{2}^{*} / \Lambda_{2}\right\}\right)$;

(2-2) if $\Lambda_{2} \leq \underline{\lambda}_{2}^{*}$ and $\Lambda_{1}+\Lambda_{2} \geq \max \left\{\hat{\lambda}, \underline{\lambda}_{1}\left(\Lambda_{2}\right)\right\}$, then $p^{E Q}=\max \left\{p_{1}^{*}, p_{1}\left(\Lambda_{1}+\Lambda_{2}\right)\right\}$ and $\left(\theta_{1}^{E Q}, \theta_{2}^{E Q}\right)=\left(\min \left\{1,\left(\lambda_{1}^{*}-\Lambda_{2}\right) / \Lambda_{1}\right\}, 1\right)$

(2-3) if $\hat{\lambda} \leq \Lambda_{2} \leq \underline{\lambda}_{2}^{*}$ and $\Lambda_{1}+\Lambda_{2} \leq \underline{\lambda}_{1}\left(\Lambda_{2}\right)$, then $p^{E Q}=p_{2}\left(\Lambda_{2}\right)$ and $\left(\theta_{1}^{E Q}, \theta_{2}^{E Q}\right)=(0,1)$;

(2-4) if $\underline{\lambda}_{2}\left(\Lambda_{1}\right) \leq \Lambda_{1}+\Lambda_{2} \leq \hat{\lambda}$, then $p^{E Q}=p_{2}\left(\Lambda_{1}+\Lambda_{2}\right)$ and $\left(\theta_{1}^{E Q}, \theta_{2}^{E Q}\right)=(1,1)$;

(2-5) if $\Lambda_{1} \leq \hat{\lambda}$ and $\Lambda_{1}+\Lambda_{2} \leq \underline{\lambda}_{2}\left(\Lambda_{1}\right)$, then $p^{E Q}=p_{1}\left(\Lambda_{1}\right)$ and $\left(\theta_{1}^{E Q}, \theta_{2}^{E Q}\right)=(1,0)$.

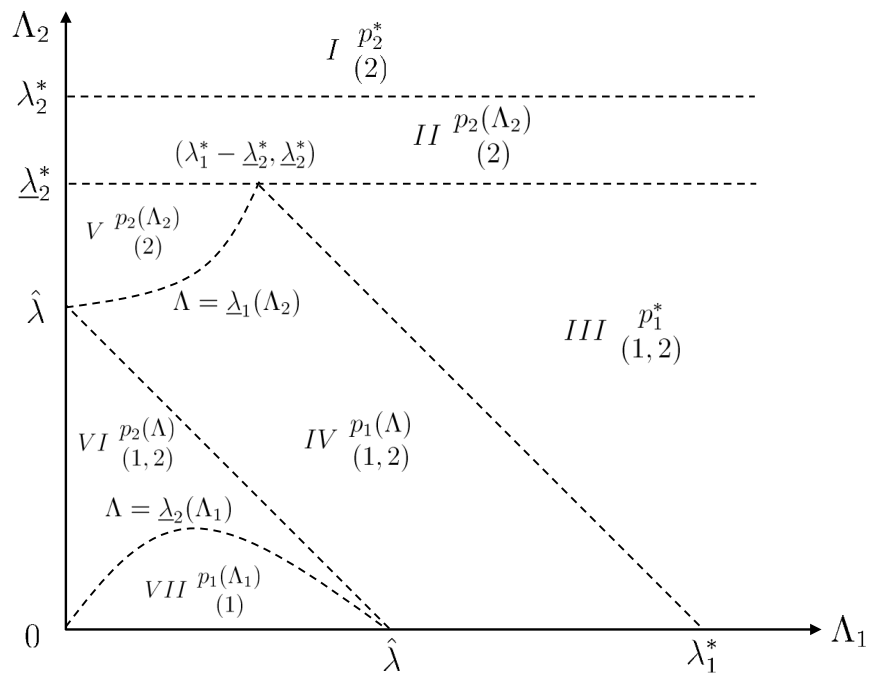

Figure 5: Optimal policy when $\hat{p} \geq \max \left\{p_{1}^{*}, p_{2}^{*}\right\}$

Case 3. $p_{2}^{*}<\hat{p}<p_{1}^{*}$ and $p_{1}^{*} \lambda_{1}^{*} \geq p_{2}^{*} \lambda_{2}^{*}$. In this case the optimal pricing strategy of the firm is given according to the following subcases, and it is illustrated in Figure 6:

(3-1) if $\Lambda_{1} \geq \underline{\lambda}_{1}^{*}$, then $p^{E Q}=\max \left\{p_{1}^{*}, p_{1}\left(\Lambda_{1}\right)\right\}$, and $\left(\theta_{1}^{E Q}, \theta_{2}^{E Q}\right)=\left(\min \left\{1, \lambda_{1}^{*} / \Lambda_{1}\right\}, 0\right)$;

(3-2) if $\Lambda_{1} \leq \underline{\lambda}_{1}^{*}$ and $\Lambda_{2} \geq \max \left\{\hat{\lambda}, \underline{\lambda}_{2}\left(\Lambda_{1}\right)\right\}$, then $p^{E Q}=\max \left\{p_{2}^{*}, p_{2}\left(\Lambda_{2}\right)\right\}$ and $\left(\theta_{1}^{E Q}, \theta_{2}^{E Q}\right)=\left(0, \min \left\{1, \lambda_{2}^{*} / \Lambda_{2}\right\}\right)$

(3-3) if $\underline{\lambda}_{1}(\hat{\lambda}) \leq \Lambda_{1} \leq \underline{\lambda}_{1}^{*}$ and $\Lambda_{2} \leq \underline{\lambda}_{2}\left(\Lambda_{1}\right)$, or $\Lambda_{1} \leq \underline{\lambda}_{1}(\hat{\lambda}), \Lambda_{2} \leq \hat{\lambda}$ and $\Lambda_{1}+\Lambda_{2} \leq \underline{\lambda}_{2}\left(\Lambda_{1}\right)$, then $p^{E Q}=p_{1}\left(\Lambda_{1}\right)$ and $\left(\theta_{1}^{E Q}, \theta_{2}^{E Q}\right)=(1,0)$;

(3-4) if $\Lambda_{1} \leq \underline{\lambda}_{1}(\hat{\lambda}), \Lambda_{2} \leq \hat{\lambda}$ and $\Lambda_{1}+\Lambda_{2} \geq \hat{\lambda}$, then $p^{E Q}=\hat{p}$ and any $\left(\theta_{1}^{E Q}, \theta_{2}^{E Q}\right)$ in the following set is an equilibrium: $\left\{\left(\theta_{1}, \theta_{2}\right): 0 \leq \theta_{1}, \theta_{2} \leq 1, \theta_{1} \Lambda_{1}+\theta_{2} \Lambda_{2}=\hat{\lambda}\right\}$; 


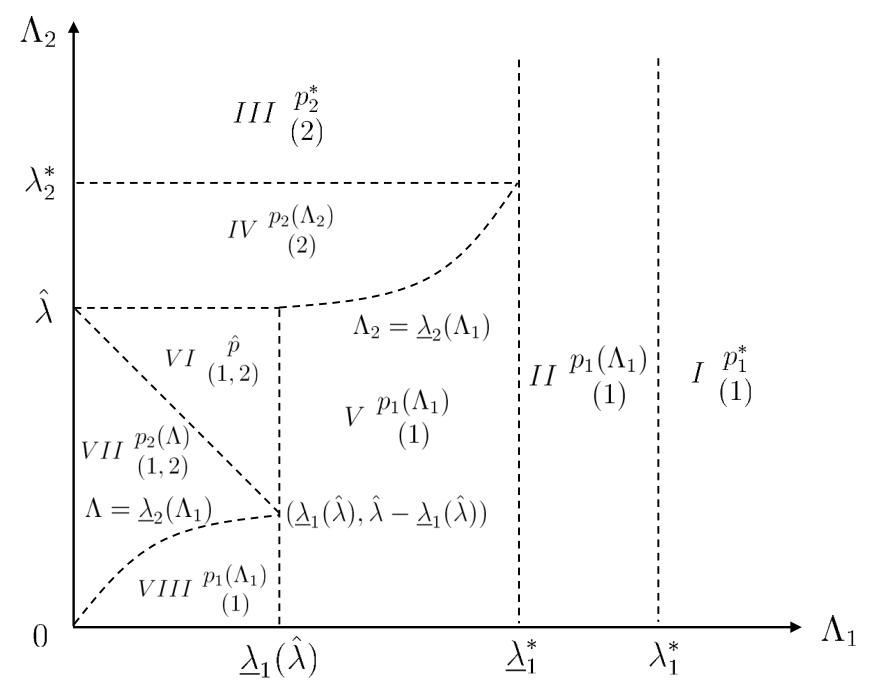

Figure 6: Optimal policy when $p_{2}^{*}<\hat{p}<p_{1}^{*}$ and $p_{2}^{*} \lambda_{2}^{*} \leq p_{1}^{*} \lambda_{1}^{*}$

(3-5) if $\Lambda_{1} \leq \underline{\lambda}_{1}(\hat{\lambda})$ and $\underline{\lambda}_{2}\left(\Lambda_{1}\right) \leq \Lambda_{1}+\Lambda_{2} \leq \hat{\lambda}$, then $p^{E Q}=p_{2}\left(\Lambda_{1}+\Lambda_{2}\right)$ and $\left(\theta_{1}^{E Q}, \theta_{2}^{E Q}\right)=(1,1)$.

Note that for clarity, in Figure 6 we have displayed Subcase (3-1) in regions (I) and (II) according to $\Lambda_{1} \geq \lambda_{1}^{*}$ and $\Lambda_{1} \leq \lambda_{1}^{*}$, respectively. Similarly, we have displayed Subcase (3-2) in regions (III) and (IV) and Subcase (3-4) in regions (IV) and (VIII).

Case 4. $p_{2}^{*}<\hat{p}<p_{1}^{*}$ and $p_{1}^{*} \lambda_{1}^{*}<p_{2}^{*} \lambda_{2}^{*}$. In this case, the optimal pricing strategy is given according to the following subcases, and it is illustrated in Figure 7 :

(4-1) if $\Lambda_{2} \geq \underline{\lambda}_{2}^{*}$, then $p^{E Q}=\max \left\{p_{2}^{*}, p_{2}\left(\Lambda_{2}\right)\right\}$ and $\left(\theta_{1}^{E Q}, \theta_{2}^{E Q}\right)=\left(0, \min \left\{1, \lambda_{2}^{*} / \Lambda_{2}\right\}\right)$;

(4-2) if $\Lambda_{2} \leq \underline{\lambda}_{2}^{*}$ and $\Lambda_{1} \geq \lambda_{1}^{*}$, then $p^{E Q}=p_{1}^{*}$ and $\left(\theta_{1}^{E Q}, \theta_{2}^{E Q}\right)=\left(\lambda_{1}^{*} / \Lambda_{1}, 0\right)$;

(4-3) if $\Lambda_{2} \leq \underline{\lambda}_{2}^{*}$ and $\Lambda_{1} \leq \underline{\lambda}_{1}^{*}=\lambda_{1}^{*}$, then this subcase can be further decomposed into five regions (IV) to (VIII) in exactly the same way as that in Case 3, and the optimal price in each region is also the same as that in the respective region in Case 3.

\section{Proof of Theorems 4 and 5.}

In the following proof. We only prove the results on the optimal price. Based on these results, the customers' equilibrium joining probabilities can be obtained straightforwardly via Theorem 2 .

By Theorem 2, it can be seen that the firm's equilibrium total arrival rate $\lambda(p)$ can be written as

$$
\lambda(p)=\left\{\begin{array}{lc}
\max \left\{\min \left\{\Lambda_{2}, \lambda_{2}(p)\right\}, \min \left\{\Lambda_{1}+\Lambda_{2}, \lambda_{1}(p)\right\}\right\}, & 0 \leq p \leq \hat{p} \\
\max \left\{\min \left\{\Lambda_{1}, \lambda_{1}(p)\right\}, \min \left\{\Lambda_{1}+\Lambda_{2}, \lambda_{2}(p)\right\}\right\}, & p \geq \hat{p}
\end{array}\right.
$$




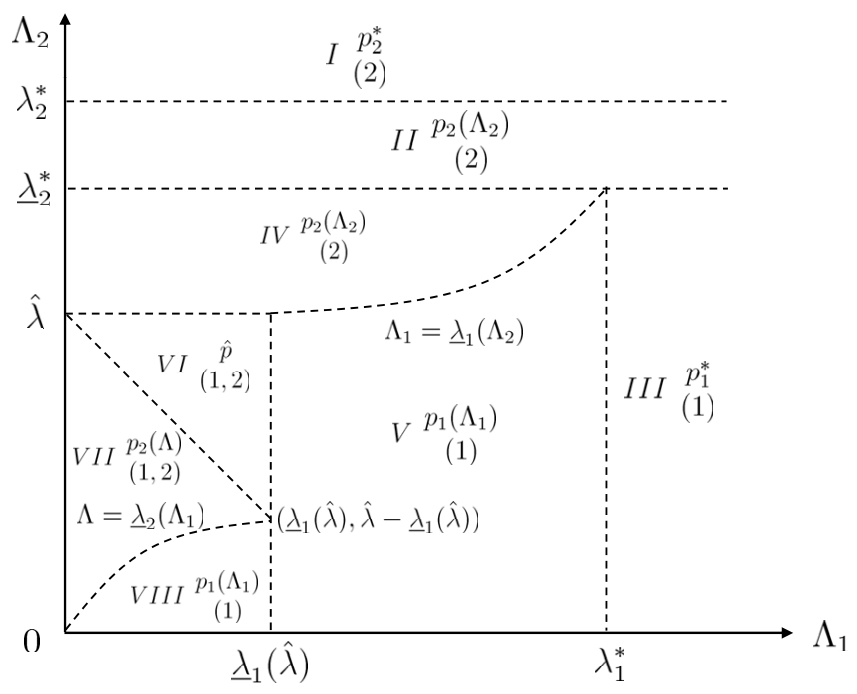

Figure 7: Optimal policy when $p_{2}^{*}<\hat{p}<p_{1}^{*}$ and $p_{2}^{*} \lambda_{2}^{*} \geq p_{1}^{*} \lambda_{1}^{*}$

Hence, the firm's revenue function $\pi(p)=p \lambda(p)$ can be written as

$$
\pi(p)=\left\{\begin{array}{lc}
\max \left\{\min \left\{\Lambda_{2} p, \pi_{2}(p)\right\}, \min \left\{\left(\Lambda_{1}+\Lambda_{2}\right) p, \pi_{1}(p)\right\}\right\}, & 0 \leq p \leq \hat{p} \\
\max \left\{\min \left\{\Lambda_{1} p, \pi_{1}(p)\right\}, \min \left\{\left(\Lambda_{1}+\Lambda_{2}\right) p, \pi_{2}(p)\right\}\right\}, & p \geq \hat{p}
\end{array}\right.
$$

Case 1. Suppose $\hat{p} \leq \min \left\{p_{1}^{*}, p_{2}^{*}\right\}$. In this case, $\pi_{1}(p)$ and $\pi_{2}(p)$ are both increasing in $p$ when $0 \leq p \leq \hat{p}$. Thus, it follows from (12) that $\max _{p \geq 0} \pi(p)=\max _{p \geq \hat{p}} \pi(p)$. Notice that the objective function $\pi(p)$ in (12) when $p \geq \hat{p}$ is exactly the same as that in (10) for the dominating customers case. In addition, by Lemma 6 , Lemma 5 continues to hold and $\tilde{\pi}_{1}\left(\lambda_{1}^{*}\right) \geq \tilde{\pi}_{2}\left(\lambda_{2}^{*}\right)$. Therefore, following the same arguments as those in the proof of Theorem 3, we can show that the firm's optimal pricing strategy for Case 1 is exactly the same as that in Theorem 3 .

Case 2. Suppose $\hat{p} \geq \max \left\{p_{1}^{*}, p_{2}^{*}\right\}$. In this case, $\pi_{1}(p)$ and $\pi_{2}(p)$ are both decreasing in $p$ when $p \geq \hat{p}$. By Lemma $3, \min \left\{\lambda_{1}^{*}, \lambda_{2}^{*}\right\} \geq \hat{\lambda}=\lambda_{1}(\hat{p})=\lambda_{2}(\hat{p})$. Then, $\pi(\hat{p})=\min \left\{\Lambda_{1}+\Lambda_{2}, \hat{\lambda}\right\} \hat{p}$. In what follows, we divide the analysis into two subcases: 1) $\Lambda_{1}+\Lambda_{2} \geq \hat{\lambda}$; and 2) $\Lambda_{1}+\Lambda_{2} \leq \hat{\lambda}$.

We first consider $\Lambda_{1}+\Lambda_{2} \geq \hat{\lambda}$. In this subcase, $\pi(\hat{p})=\hat{p} \hat{\lambda}$. When $p \geq \hat{p}$, we have $\min \left\{\Lambda_{1} p, \pi_{1}(p)\right\} \leq$ $\pi_{1}(p) \leq \pi_{1}(\hat{p})=\pi(\hat{p})$ and $\min \left\{\left(\Lambda_{1}+\Lambda_{2}\right) p, \pi_{2}(p)\right\} \leq \pi_{2}(\hat{p})=\pi(\hat{p})$. Then, by $(12), \max _{p \geq 0} \pi(p)=$ $\max _{0 \leq p \leq \hat{p}} \pi(p)$. Thus,

$$
\begin{aligned}
\max _{p \geq 0} \pi(p) & =\max \left\{\max _{0 \leq p \leq \hat{p}}\left\{\min \left\{\Lambda_{2} p, \pi_{2}(p)\right\}\right\}, \max _{0 \leq p \leq \hat{p}}\left\{\min \left\{\left(\Lambda_{1}+\Lambda_{2}\right) p, \pi_{1}(p)\right\}\right\}\right\} \\
& =\max \left\{\max _{p_{2}^{*} \leq p \leq \hat{p}}\left\{\min \left\{\Lambda_{2} p, \pi_{2}(p)\right\}\right\}, \max _{p_{1}^{*} \leq p \leq \hat{p}}\left\{\min \left\{\left(\Lambda_{1}+\Lambda_{2}\right) p, \pi_{1}(p)\right\}\right\}\right\} .
\end{aligned}
$$

Note that $\Lambda_{2} p$ is increasing in $p$ and $\pi_{2}(p)$ is strictly decreasing in $p$ when $p \geq p_{2}^{*}$. Then, when $\Lambda_{2} \geq$ $\lambda_{2}^{*}$, we have $\max _{p_{2}^{*} \leq p \leq \hat{p}}\left\{\min \left\{\Lambda_{2} p, \pi_{2}(p)\right\}\right\}=\pi_{2}\left(p_{2}^{*}\right)=p_{2}^{*} \lambda_{2}^{*}$; and when $\Lambda_{2} \leq \lambda_{2}^{*}, \min \left\{\Lambda_{2} p, \pi_{2}(p)\right\}$ 
achieves maximum over $p_{2}^{*} \leq p \leq \hat{p}$ at $p=\min \left\{p_{2}\left(\Lambda_{2}\right), \hat{p}\right\}=p_{2}\left(\max \left\{\Lambda_{2}, \hat{\lambda}\right\}\right)$. Thus, by the definition of $\tilde{\pi}_{2}(\lambda)$, we have

$$
\max _{p_{2}^{*} \leq p \leq \hat{p}}\left\{\min \left\{\Lambda_{2} p, \pi_{2}(p)\right\}\right\}=\frac{\Lambda_{2}}{\max \left\{\Lambda_{2}, \hat{\lambda}\right\}} \tilde{\pi}_{2}\left(\min \left\{\max \left\{\Lambda_{2}, \hat{\lambda}\right\}, \lambda_{2}^{*}\right\}\right) .
$$

Similarly and by noting that $\Lambda_{1}+\Lambda_{2} \geq \hat{\lambda}$, we obtain

$$
\max _{p_{1}^{*} \leq p \leq \hat{p}}\left\{\min \left\{\left(\Lambda_{1}+\Lambda_{2}\right) p, \pi_{1}(p)\right\}\right\}=\tilde{\pi}_{1}\left(\min \left\{\Lambda_{1}+\Lambda_{2}, \lambda_{1}^{*}\right\}\right) .
$$

Hence,

$$
\max _{p \geq 0} \pi(p)=\max \left\{\frac{\Lambda_{2}}{\max \left\{\Lambda_{2}, \hat{\lambda}\right\}} \tilde{\pi}_{2}\left(\min \left\{\max \left\{\Lambda_{2}, \hat{\lambda}\right\}, \lambda_{2}^{*}\right\}\right), \tilde{\pi}_{1}\left(\min \left\{\Lambda_{1}+\Lambda_{2}, \lambda_{1}^{*}\right\}\right)\right\}
$$

It can be easily verified from the above procedure that: 1) $p^{*}=p_{1}^{*}$ if $\left.\max _{p \geq 0} \pi(p)=\tilde{\pi}_{1}\left(\lambda_{1}^{*}\right) ; 2\right)$ $p^{*}=p_{1}\left(\Lambda_{1}+\Lambda_{2}\right)$ if $\left.\max _{p \geq 0} \pi(p)=\tilde{\pi}_{1}\left(\Lambda_{1}+\Lambda_{2}\right) ; 3\right) p^{*}=p_{2}^{*}$ if $\max _{p \geq 0} \pi(p)=\tilde{\pi}_{2}\left(\lambda_{2}^{*}\right)$; and $p^{*}=p_{2}\left(\Lambda_{2}\right)$ if $\max _{p \geq 0} \pi(p)=\tilde{\pi}_{2}\left(\Lambda_{2}\right)$.

We are now ready to prove $(2-1)$ to $(2-3)$. Note that by Lemma $2, \tilde{\pi}_{1}\left(\lambda_{1}^{*}\right) \leq \tilde{\pi}_{2}\left(\lambda_{2}^{*}\right)$ for Case 2 .

We first prove (2-1) and (2-3). When $\Lambda_{2} \geq \lambda_{2}^{*}, \max _{p \geq 0} \pi(p)=\tilde{\pi}_{2}\left(\lambda_{2}^{*}\right)$ by (13). Thus, the optimal price is $p_{2}^{*}$ when $\Lambda_{2} \geq \lambda_{2}^{*}$. Now suppose either $\underline{\lambda}_{2}^{*} \leq \Lambda_{2} \leq \lambda_{2}^{*}$ or $\hat{\lambda} \leq \Lambda_{2} \leq \underline{\lambda}_{2}^{*}$ and $\Lambda_{1}+\Lambda_{2} \leq \underline{\lambda}_{1}\left(\Lambda_{2}\right)$. In both cases, by (13), we have

$$
\max _{p \geq 0} \pi(p)=\max \left\{\tilde{\pi}_{2}\left(\Lambda_{2}\right), \tilde{\pi}_{1}\left(\min \left\{\Lambda_{1}+\Lambda_{2}, \lambda_{1}^{*}\right\}\right)\right\} .
$$

When $\underline{\lambda}_{2}^{*} \leq \Lambda_{2} \leq \lambda_{2}^{*}$, from the definition of $\underline{\lambda}_{2}^{*}, \tilde{\pi}_{2}\left(\Lambda_{2}\right) \geq \tilde{\pi}_{2}\left(\underline{\lambda}_{2}^{*}\right)=\pi_{1}\left(p_{1}^{*}\right)$. When $\hat{\lambda} \leq \Lambda_{2} \leq \underline{\lambda}_{2}^{*}$ and $\Lambda_{1}+\Lambda_{2} \leq \underline{\lambda}_{1}\left(\Lambda_{2}\right)$, it follows from Lemma 6 that $\Lambda_{1}+\Lambda_{2} \leq \lambda_{1}^{*}$. Then, $\tilde{\pi}_{1}\left(\min \left\{\Lambda_{1}+\Lambda_{2}, \lambda_{1}^{*}\right\}\right)=$ $\tilde{\pi}_{1}\left(\Lambda_{1}+\Lambda_{2}\right) \leq \tilde{\pi}_{1}\left(\underline{\lambda}_{1}\left(\Lambda_{2}\right)\right)=\tilde{\pi}_{2}\left(\Lambda_{2}\right)$. Thus, for both cases, $\max _{p \geq 0} \pi(p)=\tilde{\pi}_{2}\left(\Lambda_{2}\right)$, and the optimal price is $p_{2}\left(\Lambda_{2}\right)$.

We next prove (2-2). First suppose $\Lambda_{2} \leq \underline{\lambda}_{2}^{*}$ and $\Lambda_{1}+\Lambda_{2} \geq \lambda_{1}^{*}$. In this case, since $\hat{\lambda} \leq \underline{\lambda}_{2}^{*} \leq \lambda_{2}^{*}$, we have

$$
\tilde{\pi}_{2}\left(\min \left\{\max \left\{\Lambda_{2}, \hat{\lambda}\right\}, \lambda_{2}^{*}\right\}\right)=\tilde{\pi}_{2}\left(\max \left\{\Lambda_{2}, \hat{\lambda}\right\}\right) \leq \tilde{\pi}_{2}\left(\underline{\lambda}_{2}^{*}\right)=\tilde{\pi}_{1}\left(\lambda_{1}^{*}\right)=\tilde{\pi}_{1}\left(\min \left\{\Lambda_{1}+\Lambda_{2}, \lambda_{1}^{*}\right\}\right)
$$

Then, by (13), $\max _{p \geq 0} \pi(p)=\tilde{\pi}_{1}\left(\lambda_{1}^{*}\right)$. Thus, the optimal price $p_{1}^{*}$.

We next suppose $\Lambda_{2} \leq \underline{\lambda}_{2}^{*}$ and $\max \left\{\hat{\lambda}, \underline{\lambda}_{1}\left(\Lambda_{2}\right)\right\} \leq \Lambda_{1}+\Lambda_{2} \leq \lambda_{1}^{*}$. In this case, by (13),

$$
\left.\max _{p \geq 0} \pi(p)=\max \left\{\frac{\Lambda_{2}}{\max \left\{\Lambda_{2}, \hat{\lambda}\right\}} \tilde{\pi}_{2}\left(\max \left\{\Lambda_{2}, \hat{\lambda}\right\}\right), \tilde{\pi}_{1}\left(\Lambda_{1}+\Lambda_{2}\right\}\right)\right\} .
$$

When $\hat{\lambda} \leq \underline{\lambda}_{1}\left(\Lambda_{2}\right)$, from the definition of $\underline{\lambda}_{1}\left(\Lambda_{2}\right)$, we have

$$
\tilde{\pi}_{2}\left(\Lambda_{2}\right)=\tilde{\pi}_{1}\left(\underline{\lambda}_{1}\left(\Lambda_{2}\right)\right) \leq \tilde{\pi}_{1}\left(\Lambda_{1}+\Lambda_{2}\right) .
$$


When $\hat{\lambda}>\underline{\lambda}_{1}\left(\Lambda_{2}\right)$, since $\Lambda_{2} \leq \hat{\lambda} \leq \Lambda_{1}+\Lambda_{2}$, then $\tilde{\pi}_{1}\left(\Lambda_{1}+\Lambda_{2}\right) \geq \tilde{\pi}_{1}(\hat{\lambda})=\tilde{\pi}_{2}(\hat{\lambda}) \geq \tilde{\pi}_{2}\left(\Lambda_{2}\right)$. Thus, for both cases, $\max _{p \geq 0} \pi(p)=\tilde{\pi}_{1}\left(\Lambda_{1}+\Lambda_{2}\right)$, and the optimal price is $p_{1}\left(\Lambda_{1}+\Lambda_{2}\right)$.

We next consider $\Lambda_{1}+\Lambda_{2} \leq \hat{\lambda}$. In this subcase, when $0 \leq p \leq \hat{p}$, since $\lambda_{1}(p)$ and $\lambda_{2}(p)$ are decreasing in $p$ by Lemma 2 , it follows that

$$
\begin{aligned}
& \Lambda_{2} p \leq \hat{\lambda} p=\lambda_{2}(\hat{p}) p \leq \lambda_{2}(p) p=\pi_{2}(p) ; \\
&\left(\Lambda_{1}+\Lambda_{2}\right) p \leq \hat{\lambda} p=\lambda_{1}(\hat{p}) p \leq \lambda_{1}(p) p=\pi_{1}(p) .
\end{aligned}
$$

Then, by (12), $\pi(p)=\left(\Lambda_{1}+\Lambda_{2}\right) p$ is increasing in $p$ when $0 \leq p \leq \hat{p}$. Therefore, $\max _{p \geq 0} \pi(p)=$ $\max _{p \geq \hat{p}} \pi(p)$. In addition, since $\Lambda_{1} \hat{p} \leq \pi_{1}(\hat{p}),\left(\Lambda_{1}+\lambda_{2}\right) \hat{p} \leq \pi_{2}(\hat{p})$, and $\pi_{1}(p)$ and $\pi_{2}(p)$ are decreasing in $p$ when $p \geq \hat{p}$, it is straightforward to verify from (12) that

$$
\max _{p \geq 0} \pi(p)=\max \left\{\tilde{\pi}_{1}\left(\Lambda_{1}\right), \tilde{\pi}_{2}\left(\Lambda_{1}+\Lambda_{2}\right)\right\}
$$

Now we are ready to prove (2-4) and (2-5). When $\underline{\lambda}_{2}\left(\Lambda_{1}\right) \leq \Lambda_{1}+\Lambda_{2} \leq \hat{\lambda}$, we have $\tilde{\pi}_{1}\left(\Lambda_{1}\right)=$ $\tilde{\pi}_{2}\left(\underline{\lambda}_{2}\left(\Lambda_{1}\right)\right) \leq \tilde{\pi}_{2}\left(\Lambda_{1}+\Lambda_{2}\right)$ by the definition of $\underline{\lambda}_{2}\left(\Lambda_{1}\right)$ and the monotonicity of $\tilde{\pi}_{2}(x)$ over $0 \leq x \leq \lambda_{2}^{*}$; then $\max _{p \geq 0} \pi(p)=\tilde{\pi}_{2}\left(\Lambda_{1}+\Lambda_{2}\right)$ and the optimal price is $p_{2}\left(\Lambda_{1}+\Lambda_{2}\right)$. On the other hand, when $\Lambda_{1} \leq \hat{\lambda}$ and $\Lambda_{1}+\Lambda_{2} \leq \underline{\lambda}_{2}\left(\Lambda_{1}\right)$, we have $\Lambda_{1}+\Lambda_{2} \leq \hat{\lambda}$ by Lemma 6 ; and it is easy to verify that $\max _{p \geq 0} \pi(p)=\tilde{\pi}_{1}\left(\Lambda_{1}\right)$ and the optimal price is $p_{1}\left(\Lambda_{1}\right)$. The proof for Case 2 is complete.

Case 3. Suppose $p_{2}^{*}<\hat{p}<p_{1}^{*}$. Then, $\lambda_{1}^{*}<\hat{\lambda}<\lambda_{2}^{*}$ by Lemma 3. In this case, $\pi_{1}(p)$ is increasing in $p$ when $0 \leq p \leq \hat{p}$, and $\pi_{2}(p)$ is decreasing in $p$ when $p \geq \hat{p}$. Then, by (12), we have

$$
\begin{aligned}
\max _{p \geq 0} \pi(p)=\max \{ & \max _{p_{2}^{*} \leq p \leq \hat{p}}\left\{\min \left\{\Lambda_{2} p, \pi_{2}(p)\right\}\right\}, \min \left\{\Lambda_{1}+\Lambda_{2}, \hat{\lambda}\right\} \hat{p}, \\
& \left.\max _{p \geq p_{1}^{*}}\left\{\min \left\{\Lambda_{1} p, \pi_{1}(p)\right\}\right\}, \max _{p \geq \hat{p}}\left\{\min \left\{\left(\Lambda_{1}+\Lambda_{2}\right) p, \pi_{2}(p)\right\}\right\}\right\} .
\end{aligned}
$$

By similar arguments as those in the above proofs for Case 1 (or Theorem 3 ) and Case 2, it is easy to show that

$$
\begin{aligned}
\max _{p_{2}^{*} \leq p \leq \hat{p}}\left\{\min \left\{\Lambda_{2} p, \pi_{2}(p)\right\}\right\} & =\frac{\Lambda_{2}}{\max \left\{\Lambda_{2}, \hat{\lambda}\right\}} \tilde{\pi}_{2}\left(\min \left\{\max \left\{\Lambda_{2}, \hat{\lambda}\right\}, \lambda_{2}^{*}\right\}\right) ; \\
\max _{p \geq p_{1}^{*}}\left\{\min \left\{\Lambda_{1} p, \pi_{1}(p)\right\}\right\} & =\tilde{\pi}_{1}\left(\min \left\{\Lambda_{1}, \lambda_{1}^{*}\right\}\right) ; \\
\max _{p \geq \hat{p}}\left\{\min \left\{\left(\Lambda_{1}+\Lambda_{2}\right) p, \pi_{2}(p)\right\}\right\} & =\tilde{\pi}_{2}\left(\min \left\{\Lambda_{1}+\Lambda_{2}, \hat{\lambda}\right\}\right) .
\end{aligned}
$$

Substituting above identities into (14) and noting that $\tilde{\pi}_{2}\left(\min \left\{\Lambda_{1}+\Lambda_{2}, \hat{\lambda}\right\}\right) \geq \min \left\{\Lambda_{1}+\Lambda_{2}, \hat{\lambda}\right\} \hat{p}$, we obtain

$$
\begin{aligned}
\max _{p \geq 0} \pi(p)=\max \left\{\frac{\Lambda_{2}}{\max \left\{\Lambda_{2}, \hat{\lambda}\right\}}\right. & \tilde{\pi}_{2}\left(\min \left\{\max \left\{\Lambda_{2}, \hat{\lambda}\right\}, \lambda_{2}^{*}\right\}\right), \\
& \left.\tilde{\pi}_{1}\left(\min \left\{\Lambda_{1}, \lambda_{1}^{*}\right\}\right), \tilde{\pi}_{2}\left(\min \left\{\Lambda_{1}+\Lambda_{2}, \hat{\lambda}\right\}\right)\right\} .
\end{aligned}
$$


From the above procedure, it can be easily shown that: 1) $p^{*}=p_{1}^{*}$ if $\max _{p \geq 0} \pi(p)=\tilde{\pi}_{1}\left(\lambda_{1}^{*}\right)$; 2) $p^{*}=p_{1}\left(\Lambda_{1}\right)$ if $\max _{p \geq 0} \pi(p)=\tilde{\pi}_{1}\left(\Lambda_{1}\right)$; 3) $p^{*}=p_{2}^{*}$ if $\max _{p \geq 0} \pi(p)=\tilde{\pi}_{2}\left(\lambda_{2}^{*}\right)$; 4) $p^{*}=p_{2}\left(\Lambda_{2}\right)$ if $\max _{p \geq 0} \pi(p)=\tilde{\pi}_{2}\left(\Lambda_{2}\right)$; 5) $p^{*}=\hat{p}$ if $\max _{p \geq 0} \pi(p)=\tilde{\pi}_{2}(\hat{\lambda})$; and 6) $p^{*}=p_{2}\left(\Lambda_{1}+\Lambda_{2}\right)$ if $\max _{p \geq 0} \pi(p)=$ $\tilde{\pi}_{2}\left(\Lambda_{1}+\Lambda_{2}\right)$.

Now we are ready to prove the results for Case 3 . Note that $\tilde{\pi}_{1}\left(\lambda_{1}^{*}\right) \geq \tilde{\pi}_{2}\left(\lambda_{2}^{*}\right)$ in Case 3 .

(3-1) When $\Lambda_{1} \geq \lambda_{1}^{*}$, then $\max _{p \geq 0} \pi(p)=\tilde{\pi}_{1}\left(\lambda_{1}^{*}\right)$ by (15). Thus, the optimal price is $p_{1}^{*}$ when $\Lambda_{1} \geq \lambda_{1}^{*}$. Now suppose $\underline{\lambda}_{1}^{*} \leq \Lambda_{1} \leq \lambda_{1}^{*}$. Then, from the definition of $\underline{\lambda}_{1}^{*}, \tilde{\pi}_{1}\left(\Lambda_{1}\right) \geq \tilde{\pi}_{2}\left(\underline{\lambda}_{1}^{*}\right)=\pi_{2}\left(p_{2}^{*}\right)$. Thus, $\max _{p \geq 0} \pi(p)=\tilde{\pi}_{1}\left(\Lambda_{1}\right)$ by $(15)$, and the optimal price is $p_{1}\left(\Lambda_{1}\right)$.

(3-2) First suppose $0 \leq \Lambda_{1} \leq \underline{\lambda}_{1}^{*}$ and $\Lambda_{2} \geq \lambda_{2}^{*}$. In this case, since $\underline{\lambda}_{1}^{*} \leq \lambda_{1}^{*}$, we have

$$
\tilde{\pi}_{1}\left(\min \left\{\Lambda_{1}, \lambda_{1}^{*}\right\}\right)=\tilde{\pi}_{1}\left(\Lambda_{1}\right) \leq \tilde{\pi}_{1}\left(\underline{\lambda}_{1}\right)=\tilde{\pi}_{2}\left(\lambda_{2}^{*}\right)=\tilde{\pi}_{2}\left(\min \left\{\Lambda_{2}, \lambda_{2}^{*}\right\}\right) .
$$

Then, by (15), $\max _{p \geq 0} \pi(p)=\tilde{\pi}_{2}\left(\lambda_{2}^{*}\right)$. Thus, the optimal price is $p_{2}^{*}$.

Now suppose $\underline{\lambda}_{1}(\hat{\lambda}) \leq \Lambda_{1} \leq \underline{\lambda}_{1}^{*}$ and $\underline{\lambda}_{2}\left(\Lambda_{1}\right) \leq \Lambda_{2} \leq \lambda_{2}^{*}$, or, $\Lambda_{1} \leq \underline{\lambda}_{1}(\hat{\lambda})$ and $\hat{\lambda} \leq \Lambda_{2} \leq \lambda_{2}^{*}$. In both subcases, $\hat{\lambda} \leq \Lambda_{2} \leq \lambda_{2}^{*}$ and $\Lambda_{1} \leq \Lambda_{1}^{*}$ by Lemma 6 . Then, by (15), we have

$$
\max _{p \geq 0} \pi(p)=\max \left\{\tilde{\pi}_{2}\left(\Lambda_{2}\right), \tilde{\pi}_{1}\left(\Lambda_{1}\right)\right\} .
$$

For the first case, by the definition of $\underline{\lambda}_{2}\left(\Lambda_{1}\right), \tilde{\pi}_{1}\left(\Lambda_{1}\right)=\tilde{\pi}_{2}\left(\underline{\lambda}_{2}\left(\Lambda_{1}\right)\right) \leq \pi_{2}\left(\Lambda_{2}\right)$; and for the second case, $\tilde{\pi}_{1}\left(\Lambda_{1}\right) \leq \tilde{\pi}_{1}\left(\underline{\lambda}_{1}(\hat{\lambda})\right)=\pi_{2}(\hat{\lambda}) \leq \pi_{2}\left(\Lambda_{2}\right)$. Thus, for both cases, $\max _{p \geq 0} \pi(p)=\tilde{\pi}_{2}\left(\Lambda_{2}\right)$, and the optimal price is $p_{2}\left(\Lambda_{2}\right)$.

(3-3) Suppose $\underline{\lambda}_{1}(\hat{\lambda}) \leq \Lambda_{1} \leq \underline{\lambda}_{1}^{*}$ and $\Lambda_{2} \leq \underline{\lambda}_{2}\left(\Lambda_{1}\right)$, or, $\Lambda_{1} \leq \underline{\lambda}_{1}(\hat{\lambda}), \Lambda_{2} \leq \hat{\lambda}$ and $\Lambda_{1}+\Lambda_{2} \leq \underline{\lambda}_{2}\left(\Lambda_{1}\right)$. For both cases, $\tilde{\pi}_{2}\left(\min \left\{\max \left\{\Lambda_{2}, \hat{\lambda}\right\}, \Lambda_{2}^{*}\right\}\right)=\tilde{\pi}_{2}\left(\max \left\{\Lambda_{2}, \hat{\lambda}\right\}\right)$ and $\tilde{\pi}_{1}\left(\min \left\{\Lambda_{1}, \lambda_{1}^{*}\right\}\right)=\tilde{\pi}_{1}\left(\Lambda_{1}\right)$ by Lemma 6 . In addition, for the first case, since $\underline{\lambda}_{2}\left(\Lambda_{1}\right) \geq \hat{\lambda}$ by Lemma 6 , we have

$$
\tilde{\pi}_{1}\left(\Lambda_{1}\right)=\tilde{\pi}_{2}\left(\underline{\lambda}_{2}\left(\Lambda_{1}\right)\right) \geq \tilde{\pi}_{2}\left(\max \left\{\Lambda_{2}, \hat{\lambda}\right\}\right) \geq \tilde{\pi}_{2}(\hat{\lambda}) \geq \tilde{\pi}_{2}\left(\min \left\{\Lambda_{1}+\Lambda_{2}, \hat{\lambda}\right\}\right)
$$

For the second case, since $\Lambda_{2}+\Lambda_{2} \leq \hat{\lambda}$ by Lemma 6 , it holds that

$$
\tilde{\pi}_{1}\left(\Lambda_{1}\right)=\tilde{\pi}_{2}\left(\underline{\lambda}_{2}\left(\Lambda_{1}\right)\right) \geq \tilde{\pi}_{2}\left(\min \left\{\Lambda_{1}+\Lambda_{2}, \hat{\lambda}\right\}\right) \geq\left(\Lambda_{1}+\Lambda_{2}\right) \hat{p} \geq \frac{\Lambda_{2}}{\max \left\{\Lambda_{2}, \hat{\lambda}\right\}} \tilde{\pi}_{2}(\hat{\lambda}) .
$$

Thus, for both cases, $\max _{p \geq 0} \pi(p)=\tilde{\pi}_{1}\left(\Lambda_{1}\right)$ by (15), and the optimal price is $p_{1}\left(\Lambda_{1}\right)$.

(3-4) Suppose $\Lambda_{1} \leq \underline{\lambda}_{1}(\hat{\lambda})$ and $\Lambda_{2} \leq \hat{\lambda} \leq \Lambda_{1}+\Lambda_{2}$. Then, by the definition of $\underline{\lambda}_{1}(\hat{\lambda})$, we have $\tilde{\pi}_{2}(\hat{\lambda})=\tilde{\pi}_{1}\left(\underline{\lambda}_{1}(\hat{\lambda})\right) \geq \tilde{\pi}_{1}\left(\Lambda_{1}\right)=\tilde{\pi}_{1}\left(\min \left\{\Lambda_{1}, \lambda_{1}^{*}\right\}\right)$ and

$$
\frac{\Lambda_{2}}{\max \left\{\Lambda_{2}, \hat{\lambda}\right\}} \tilde{\pi}_{2}\left(\min \left\{\max \left\{\Lambda_{2}, \hat{\lambda}\right\}, \lambda_{2}^{*}\right\}\right)=\frac{\Lambda_{2}}{\hat{\lambda}} \tilde{\pi}_{2}(\hat{\lambda}) \leq \tilde{\pi}_{2}(\hat{\lambda})=\tilde{\pi}_{2}\left(\min \left\{\Lambda_{1}+\Lambda_{1}, \hat{\lambda}\right\}\right) .
$$

Thus, $\max _{p \geq 0} \pi(p)=\tilde{\pi}_{2}(\hat{\lambda})$ by $(15)$, and the optimal price is $\hat{p}$.

(3-5) Suppose $\Lambda_{1} \leq \underline{\lambda}_{1}(\hat{\lambda})$ and $\underline{\lambda}_{2}\left(\Lambda_{1}\right) \leq \Lambda_{1}+\Lambda_{2} \leq \hat{\lambda}$. In this case, by the definition of $\underline{\lambda}_{2}\left(\Lambda_{1}\right)$ and Lemma 6 , we have

$$
\tilde{\pi}_{2}\left(\min \left\{\Lambda_{1}+\Lambda_{2}, \hat{\lambda}\right\}\right)=\tilde{\pi}_{2}\left(\Lambda_{1}+\Lambda_{2}\right) \geq \tilde{\pi}_{2}\left(\underline{\lambda}_{2}\left(\Lambda_{1}\right)\right)=\tilde{\pi}_{1}\left(\Lambda_{1}\right)=\tilde{\pi}_{1}\left(\min \left\{\Lambda_{1}, \lambda_{1}^{*}\right\}\right)
$$


and

$$
\frac{\Lambda_{2}}{\max \left\{\Lambda_{2}, \hat{\lambda}\right\}} \tilde{\pi}_{2}\left(\min \left\{\max \left\{\Lambda_{2}, \hat{\lambda}\right\}, \lambda_{2}^{*}\right\}\right)=\Lambda_{2} \hat{p} \leq\left(\Lambda_{1}+\Lambda_{2}\right) \hat{p} \leq \tilde{\pi}_{2}\left(\Lambda_{1}+\Lambda_{2}\right) .
$$

Thus, $\max _{p \geq 0} \pi(p)=\tilde{\pi}_{2}\left(\Lambda_{1}+\Lambda_{2}\right)$ by (15), and the optimal price is $p_{2}\left(\Lambda_{1}+\Lambda_{2}\right)$.

Case 4. The proof of this case is essentially the same as that of Case 3, hence it is omitted.

For the comparison of the optimal revenues, we also report the different cases together in the following proposition.

Proposition 4. a) The firm's optimal revenues in different regions of Case 2 in Theorem 5 satisfy

a-1) $R^{(I)}>R^{(I I)}>R^{(I I I)}>R^{(k)}>R^{(V I)}$; and

a-2) $R^{(k)}>R^{(V I I)}, k=I V, V$.

b) The firm's optimal revenues in different regions of Cases 3 and 4 in Theorem 5 satisfy

b-1) $R^{(I)}>R^{(I I)}>R^{(I I I)}>R^{(k)}>R^{(V I)}>R^{(V I I)}, k=I V, V$; and

b-2) $R^{(V I)}>R^{(V I I I)}$.

\section{Proof of Propositions 3 and 4.}

a) When $\hat{p} \geq \max \left\{p_{1}^{*}, p_{2}^{*}\right\}$, by Lemma 6 , we have $\hat{\lambda} \leq \underline{\lambda}_{2}^{*} \leq \lambda_{1}^{*}$. Since $\tilde{\pi}_{1}(\cdot)$ and $\tilde{\pi}_{2}(\cdot)$ are strictly concave functions and by the definition of $\underline{\lambda}_{2}^{*}$, it follows that

$$
\left.R^{(V I)}<\tilde{\pi}_{1}(\hat{\lambda})<R^{(I V}\right)<\tilde{\pi}_{1}\left(\lambda_{1}^{*}\right)=R^{(I I I)}=\tilde{\pi}_{2}\left(\underline{\lambda}_{2}^{*}\right)<R^{(I I)}<\tilde{\pi}_{2}\left(\lambda_{2}^{*}\right)=R^{(I)},
$$

and $R^{(V I I)}<\tilde{\pi}_{2}(\hat{\lambda})<R^{(V)}<\tilde{\pi}_{2}\left(\underline{\lambda}_{2}^{*}\right)=R^{(I I I)}$. Furthermore, from the definition of $\hat{\lambda}$ and Lemma 6 , we have $\tilde{\pi}_{1}(\hat{\lambda})=\tilde{\pi}_{2}(\hat{\lambda})$. Summarizing these results, we obtain a).

b) Suppose $p_{2}^{*}<\hat{p}<p_{1}^{*}$ and $p_{2}^{*} \lambda_{2}^{*} \leq p_{1}^{*} \lambda_{1}^{*}$. By Lemma $6, \hat{\lambda} \leq \lambda_{2}^{*}$ and $\underline{\lambda}_{1}(\hat{\lambda})<\underline{\lambda}_{1}^{*} \leq \lambda_{1}^{*}$. Since $\tilde{\pi}_{1}(\cdot)$ and $\tilde{\pi}_{2}(\cdot)$ are strictly concave functions and by the definition of $\underline{\lambda}_{1}^{*}$, it follows that

$$
R^{(V I I)}<R^{(V I)}=\tilde{\pi}_{2}(\hat{\lambda})<R^{(I V)}<\tilde{\pi}_{2}\left(\lambda_{2}^{*}\right)=R^{(I I I)}=\tilde{\pi}_{1}\left(\underline{\lambda}_{1}^{*}\right)<R^{(I I)}<\tilde{\pi}_{1}\left(\lambda_{1}^{*}\right)=R^{(I)},
$$

and $R^{(V I I I)}<\tilde{\pi}_{1}\left(\underline{\lambda}_{1}(\hat{\lambda})\right)<R^{(V)}<\tilde{\pi}_{1}\left(\underline{\lambda}_{1}^{*}\right)$. By the definition of $\hat{\lambda}$ and $\underline{\lambda}_{1}(\cdot)$, we have $\tilde{\pi}_{1}\left(\underline{\lambda}_{1}(\hat{\lambda})\right)=$ $\tilde{\pi}_{2}(\hat{\lambda})$. Summarizing these results, we obtain the results for Case 3.

The proof of the results for Case 4 is similar to those of Case 3, hence it is omitted. 